\title{
Electrokinetic Hydrogen Generation from Liquid Water
}

\section{Microjets}

\author{
Andrew M. Duffin and Richard J. Saykally* \\ Department of Chemistry, University of California, Berkeley, CA 94720 \\ and \\ Chemical Sciences Division, Lawrence Berkeley National Laboratory, Berkeley, Ca 94618
}

Author email: saykally@berkeley.edu

Address: Department of Chemistry

University of California at Berkeley

Berkeley, CA 94720

Phone : (510) 642-8269

Abstract 
We describe a method for generating molecular hydrogen directly from the charge separation effected via rapid flow of liquid water through a metal orifice, wherein the input energy is the hydrostatic pressure times the volume flow rate. Both electrokinetic currents and hydrogen production rates are shown to follow simple equations derived from the overlap of the fluid velocity gradient and the anisotropic charge distribution resulting from selective adsorption of hydroxide ions to the nozzle surface. Pressure-driven fluid flow shears away the charge balancing hydronium ions from the diffuse double layer and carries them out of the aperture. Downstream neutralization of the excess protons at a grounded target electrode produces gaseous hydrogen molecules. The hydrogen production efficiency is currently very low (ca. 106) for a single cylindrical jet, but can be improved with design changes.

\section{Keywords}

streaming current, metal-water interface, microchannel 


\section{Introduction}

The increasing financial and environmental costs of fossil fuel usage have stimulated a major campaign to develop economical alternative energy sources. Hydrogen is envisioned to become a major component of world energy within the $21^{\text {st }}$ century due to its high combustion efficiency, nonpolluting chemistry, and renewability. While several major technical problems remain to be solved, the principal obstacle to widespread implementation of "The Hydrogen Economy" is arguably the high cost of production. ${ }^{1}$ There are about 90 currently available hydrogen production routes that can be classified into biological, chemical, electrochemical, and thermal categories. ${ }^{2}$ Most hydrogen is presently made through coal gasification and by steam reformation of natural gas. These thermal methods are relatively cheap, but they do not mitigate difficulties associated with declining world petroleum reserves. Electrochemical hydrogen production methods are quite advanced and straightforward, but are currently very expensive $\left(4.5-5.5 \mathrm{kWh} / \mathrm{m}^{3} \mathrm{H}_{2}\right) .^{2}$

This paper describes a method for the production of molecular hydrogen from liquid water by exploiting the electrokinetic charge separation that can be effected in fast-flowing liquid microjets. The requisite apparatus is very simple, and involves no moving parts. The input energy is a hydrostatic pressure source and the hydrogen is produced by potential-driven reduction of water enriched in protons. Proton-enriched water is obtained via the electrical charge separation effected by rapid flow of liquid water through a metal orifice. The electrokinetic charge separation process also generates electrical power, which could be harnessed for further electrochemical water splitting. The overall efficiency of the electrokinetic power generation process (chemical + electrical) is currently ca. $10^{-3}$ for a single jet orifice, but this may be improved with design considerations listed below.

The principle of electrokinetic current generation is well known. ${ }^{3-6}$ By rapidly flowing partially ionized liquids, e.g. water, through a metal orifice, charges can be separated and transported in the liquid to create an electrokinetic (streaming) current. Early in the $20^{\text {th }}$ century, Dolezalek investigated the electrification of benzene in metal pipes. ${ }^{5,7}$ By mid-century, streaming currents were identified as the cause of otherwise mysterious petroleum industry explosions. ${ }^{5,8}$ More recently, Kwok and others have used streaming currents to generate electrical power, ${ }^{9-11}$ with Kwok describing the design of an "electrokinetic microchannel battery." In the present study, streaming currents are used to convert hydrostatic pressure directly into both electrical energy as well as stored chemical energy in the form of molecular hydrogen. 
Near the metal-water interface, selective adsorption of one type of charge carrier (hydroxide, in the case of pure water) ${ }^{12-15}$ to the metal nozzle surface creates a potential (zeta potential). To maintain charge neutrality, counterions (hydrated protons, in pure water) generate a diffuse layer of charge near the liquidsolid interface. The rapid flow of water through the metal nozzle sweeps away the diffuse, mobile layer, such that the emerging liquid water jet is positively charged via the unbalanced proton concentration. Figure 1 depicts the electrical double layer (EDL) at the unbiased metal nozzle-water interface as well as the overlap of the EDL with the velocity profile of the flowing water. Physically, it is the overlap of the charge distribution and velocity profile near the solid-liquid interface that is responsible for the streaming current. Quantitatively, streaming currents in a circular channel of radius R are described by the integral of the velocity profile, $v(r)$, and the net charge distribution,_(r), where fluid velocity and charge distribution are both functions of the radial distance, $r$, from the interface. Both v(r) and _(r) can be approximated with standard models as outlined below. ${ }^{3-6,8,16,17}$

$$
I_{s}=\int_{0}^{R} v(r) \rho(r) d r
$$

The anisotropic charge distribution is the result of the formation of an electrical double layer (EDL) at the metal nozzle-water interface. For a charged or biased surface surrounded by an electrolyte, i.e. an electrochemical electrode, the EDL forms as ions of opposite sign are attracted to the surface while ions of the same sign are correspondingly repelled. However, at an uncharged surface, the EDL is formed as a result of preferential adsorption of one ion over another. In the absence of any electron transfer reactions, overall charge neutrality is maintained across the EDL. Due to thermal fluctuations, these counterions do not remain localized near the surface and form the diffuse component of the double layer. Electrostatic interactions contract the diffuse layer, while thermal motion tends to expand it. As a result, ions are arranged according to a Boltzmann distribution

$$
n_{i}(r)=n_{0(i)} e^{\left(-z_{i} e_{c} \psi(r) / k_{b} T\right)}
$$

where $n_{o}$ is the bulk solution ion number density, $z_{i}$ is the valency and associated sign of ion $i, e_{c}$ is the fundamental charge constant, _ is the position-dependent potential, $\mathrm{k}_{\mathrm{b}}$ is Boltzmann's constant, and $\mathrm{T}$ is the absolute temperature. The net charge density _ at a given point is the sum of the individual ion number densities 


$$
\rho(r)=e_{c} \sum_{i} z_{i} n_{i}(r)
$$

For pure water, a very weak 1:1 electrolyte, $\mathrm{n}_{\mathrm{o}(+)}=\mathrm{n}_{\mathrm{o}(-)}=\mathrm{n}_{\mathrm{o}}$ with $\mathrm{n}_{\mathrm{o}}$ being governed by the autoionization constant of water. At $298 \mathrm{~K}, \mathrm{n}_{0}$ has the familiar value of $1.0 \times 10^{-7} \mathrm{~mole} / \mathrm{L}$ or $6.02 \times 10^{13} \mathrm{~cm}^{-3}$. The position dependent charge density of water is given by

$$
\rho(r)=e_{c}\left(n_{+}-n_{-}\right)=e_{c}\left[n_{o} e^{\left(-e_{c} \psi(r) / k_{b} T\right)}-n_{o} e^{\left(e_{c} \psi(r) / k_{b} T\right)}\right]=-2 e_{c} n_{o} \sinh \left(e_{c} \psi(r) / k_{b} T\right) .
$$

Poisson's equation can then be used to relate the position-dependent potential and the charge distribution

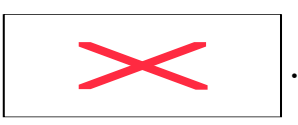

Here _ is permittivity of the medium times the permittivity of free space. Combining Equations 4 and 5 , while making a flat plate approximation (change from radial coordinate, $r$, to linear coordinate, $\mathrm{x}$ ) as well as the Debye-Huckel approximation, leads to a differential equation for the potential as a function of position:

$$
\frac{d^{2} \psi(x)}{d x^{2}}=\kappa^{2} \psi(x)
$$

_ is the inverse Debye length, viz. the inverse of the characteristic double layer thickness:

$$
\kappa=\sqrt{\frac{2 e_{c}^{2} n_{o}}{\varepsilon k_{b} T}}
$$

This simple differential equation for the potential can be readily solved and implemented to yield the net charge density distribution,_(x).

Considering the limited thickness of the aperture, the position dependent velocity $\mathrm{v}(\mathrm{x})$ can be easily modeled with a "top hat" profile. In micro-channels, the Reynolds number, defined as

$$
R_{e}=\frac{\bar{v} \cdot d \cdot \rho_{w}}{\eta_{w}}
$$

where $\bar{v}$ is the average bulk velocity, $\mathrm{d}$ is the aperture diameter, ${ }_{-\mathrm{w}}$ is the fluid density, and ${ }_{-\mathrm{w}}$ is the fluid viscosity, typically remains below the commonly accepted value of 2300 for incipient turbulent flow. However, under fast flowing conditions this criterion may not always hold true. Fortunately, we do not need to specifically consider laminar vs. turbulent flow regimes here because the situation is simplified due to the small thickness of the Pt/Ir aperture that creates the liquid jet. At the actual orifice, the Pt/Ir disk (electron microscope aperture: Ted Pella Inc.) thickness is between $0.09-0.12 \mathrm{~mm}$. For both laminar and 
turbulent flow regimes, Equations 9a and 9b give the characteristic length, L, over which developed flow can be expected to form: ${ }^{18}$

$$
\frac{L}{d} \approx 0.06 R_{e} \quad \text { (laminar)(a) } \quad \frac{L}{d} \approx 4.4 R_{e}^{1 / 6}(\text { turbulent })(\mathrm{b})
$$

For all aperture diameters and fluid velocities measured here, the aperture thickness was insufficient to develop either completely turbulent or laminar flow. Consequently, entrance effects dominate, engendering "top hat" velocity profile characteristics. Figure 1b shows the velocity profile near the metal-water interface. The fluid velocity at the water-metal interface is zero and there is a laminar sub-layer near the wall. The fluid velocity increases linearly across this laminar sublayer until it reaches bulk fluid velocity. Equation 10 describes a gradient used to model the slope of the velocity increase near the interface $:^{3,16,19,20}$

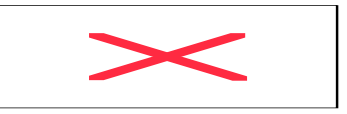

Here $\bar{v}$ is the average bulk velocity and _ $\mathrm{x}$ is a measure of the laminar sublayer thickness (_x $=116 \_\mathrm{R} \_\mathrm{R}_{\mathrm{e}}{ }^{-}$ ${ }^{7 / 8)}$ ). Figure $1 \mathrm{~b}$ also depicts the overlap of the net charge density with the velocity profile.

Combining the equations for net charge density and velocity profile into Equation 1 and integrating with the appropriate boundary conditions leads to a compact equation for the streaming current: ${ }^{3}$

$$
I_{s 1}=\frac{-2 \pi R \varepsilon \bar{v} \zeta}{\delta x}
$$

where the surface potential ${ }_{-s}$ has been replaced by the potential at the shear plane _, i.e. the potential at the position of the immobile adsorbed layer rather than at the metal surface itself. Plugging in the constants

for pure water, the streaming current equation reduces to $I_{s}=-6.8 \cdot 10^{-6} \cdot \zeta \cdot d^{0.875} \cdot \bar{v}^{1.875} \mathrm{Amps}$ where all units have been previously combined to yield amps.

\section{Experimental}

Liquid microjets are generated by using a Jasco PU-2089 HPLC pump to supply pressurized water to a jet nozzle. The jet nozzle apparatus is stainless steel and consists of a base unit and a compression disk, each with mm-scale orifices. The micron-sized jet aperture is positioned over the base orifice and sealed by tightening the compression disk to the base. Jet apertures are platinum/iridium electron microscope apertures (5-20 _m diameter) purchased form Ted Pella Inc. Water is fed to the nozzle through PEEK tubing that is vacuum sealed across a 2 _" Conflat flange. The nozzle unit is electrically isolated on the 
vacuum side of the flange. Flow rates from the pump range from 0-3 $\mathrm{mL} / \mathrm{min}$ with pressures from 0-48 $\mathrm{MPa}(\mathrm{ca} .0-500 \mathrm{~atm})$. Jet velocities are calculated from the volume flow rate in conjunction with nominal aperture diameter $\left(\right.$ velocity $(\mathrm{m} / \mathrm{s})=$ flow $\left(\mathrm{m}^{3} / \mathrm{s}\right) \div$ area $\left.\left(\mathrm{m}^{2}\right)\right)$. The water in all experiments is Milliporefiltered with a resistivity of $18.2 \mathrm{M}_{-} \mathrm{cm} . \mathrm{D}_{2} \mathrm{O}\left(99.9 \% \mathrm{D}\right.$ at $\left.0.77 \mathrm{M}_{-} \mathrm{cm}\right)$ for all requisite experiments is purchased from Cambridge Isotope Laboratories Inc. and is used without further purification. Both $\mathrm{H}_{2} \mathrm{O}$ and $\mathrm{D}_{2} \mathrm{O}$ are nitrogen-purged and degassed prior to introduction into the HPLC pump.

Streaming currents are measured by connecting the nozzle to electrical ground through a Keithley 428 Current Amplifier. The amplifier output voltage is read from a digital multimeter. Current is measured as a function of jet velocity by changing the volumetric flow rate, and consequently the backing pressure, at the HPLC pump. Streaming currents can be measured both in ambient air and in vacuum. When measurements are made in air, current can be measured both at the nozzle as well as at an isolated copper target in the path of the jet. When current is measured at the target, it is equal in magnitude but opposite in sign to the current at the nozzle.

In order to measure hydrogen production, the streaming current experiments are conducted in vacuum. The experimental setup, diagramed in Figure 2, consists of a jet chamber and an analysis chamber connected by a Varian precision leak valve. The Conflat flange with the isolated jet nozzle is attached to the top of the jet chamber. The liquid jet from the nozzle travels vertically downward $\sim 1 \mathrm{~m}$ in vacuum before striking a sealed off half-nipple, which is immersed in liquid nitrogen to cryotrap liquid water. Three inline liquid nitrogen traps separate the main part of the jet chamber from the leak valve. A Leybold-Heraeus turbo pump ( $\sim 401 / \mathrm{s})$ is used to pump the jet chamber. The three in-line liquid nitrogen traps effectively remove all water and other condensables before they reach the analysis chamber. Hydrogen produced in the jet chamber can traverse the cryotraps in diffusing to the analysis chamber. The pressure above the jet is in the sub-millitorr range and drops across the in-line traps to $\sim 10^{-6}$ torr near the leak valve. To avoid the complicating influence of hot filaments, neither ion gauge nor thermocouple pressure sensors are used during experimental runs.

The analysis chamber contains a Hiden Analytical PSM003 quadrupole mass spectrometer used for residual gas analysis and this chamber is pumped by a BOC Edwards turbopump ( 70 1/s) that maintains a base pressure of $2 * 10^{-8}$ torr. Gases from the jet chamber are leaked into the analysis chamber for mass separation and detection. Before mass separation, molecules are ionized with $70 \mathrm{eV}$ electrons from the mass spectrometer's internal ionization filament. The mass spectrometer is set for multiple ion detection 
and repeatedly scans specified masses. A typical scan cycles between masses 2, 3, 4, 18, 19, and $20\left(\mathrm{H}_{2}{ }^{+}\right.$, $\mathrm{HD}^{+}, \mathrm{D}_{2}{ }^{+}, \mathrm{H}_{2} \mathrm{O}^{+}, \mathrm{HDO}^{+}$, and $\mathrm{D}_{2} \mathrm{O}^{+}$respectively). Each ion is collected on a Secondary Electron Multiplier (SEM) for $100 \mathrm{~ms}$ and the signal is then scaled to output counts per second. The ion signals are also adjusted to account for differences in electron impact cross section. Counts/s for each charge to mass ratio are plotted against the time at which each measurement was collected.

For hydrogen generation experiments, the composition $\left(\mathrm{H}_{2} \mathrm{O} / \mathrm{D}_{2} \mathrm{O}\right)$ and the velocity of the liquid jet are varied while the mass spectrometer collects data for the specified masses. An electrical feedthrough allows for simultaneous measurement of the current at the nozzle. The relatively large $\mathrm{H}_{2}$ background present in any UHV chamber interferes with the detection of $\mathrm{H}_{2}$ generated by the liquid microjet. However, there is no corresponding $\mathrm{D}_{2}$ or $\mathrm{HD}$ background and $\mathrm{D}_{2} \mathrm{O}$ jets can be employed to effectively characterize electrokinetically generated hydrogen.

\section{Results and Discussion}

Figure 3 shows the results for streaming current measurements from a 5 _m diameter water jet running in air, with Equation 11 used to fit the experimental data. Although the fit to the experimental data is excellent, the _ potential is not faithfully reproduced between different jets and apertures. However, it should be noted that these experiments are not well-suited to obtain an accurate measure of the zeta potential for a water-Pt/Ir interface. Unfortunately, large uncertainties in aperture diameter, and therefore velocity, require the zeta potential to be treated as a fitting parameter rather than as a meaningful physical measurement.

Gavis and Kosman as well as Faubel indicate that, ${ }^{5,21}$ under dynamic conditions, the distance over which charge separation exists is contained within the laminar sublayer; that is, the charge density distribution used to derive the streaming current equation may not be rigorously correct. It is unclear whether or not the double layer thickness is contracted under dynamic conditions, or whether it overestimated even under static conditions, but in either case, it is unlikely that the EDL extends beyond the laminar sublayer adjacent to the metal-water interface. However, since the value for the zeta potential is adjusted to fit the experimental data, it is also adjusted to compensate for any charge distribution contraction.

A thinner double layer or double layer contraction can also be invoked to justify the use of the flat plate approximation rather than cylindrical coordinates. For the present conditions, the radius of curvature of all $\operatorname{apertures}\left(>5 \_\mathrm{m}\right)$ is likely to be large compared to the contracted or true double layer thickness $\left(<1 \_\mathrm{m}\right)$. 
As attempts to fit the streaming current data using cylindrical coordinates failed to yield an improvement, a one dimensional model is presumed adequate in describing the charge distribution. Although the structure of Equation 11 was first derived for more macroscopic fluid flows, it faithfully reproduces our experimental data for liquid water microjets. In spite of the difficulties in measuring the actual zeta potential, Equation 11, as well as analogous equations using similar velocity profiles, fit the experimental data very well and confirm that the streaming current scales nearly quadratically with flow velocity.

It should be noted that Equation 11 is independent of channel length. This observation is consistent with the fact that the timescale for double layer formation $(<\mathrm{ns})$ is much faster than the time it takes the water to traverse the channel ( _s) $)^{5,22}$ i.e. the excess charge shorn from the interface builds up and reaches a steady level very early in the channel. The excess ions in the fluid flow are able to equilibrate with the EDL further down the channel to inhibit further electrification and preclude any streaming current length dependence.

When pure water is used as the electrolytic solution, streaming currents are inevitably produced from the separation of hydronium (or other forms of the hydrated proton) and hydroxide ions. As the current from ground to nozzle is positive, the zeta potential must be negative, with excess negative charge existing at the metal/water interface. Thus, it is the hydroxide ions that preferentially collect at the interface, with excess hydronium ions in the diffuse layer. Fluid movement carries hydronium ions downstream and concentrates hydroxide ions in the nozzle. The excess charge creates a potential in the nozzle that induces these hydroxide ions to discharge at the Pt/Ir interface. Anion discharge at the interface forces electrons from the nozzle to electrical ground and gives rise to the positive currents observed. The liquid emerging from the aperture is positively charged due to excess hydronium ions. When this charged liquid beam encounters a grounded metal target electrode downstream, electrons flow from the electrode into the solution, reduce the hydronium, and generate hydrogen, according to the process:

$$
2 \mathrm{H}^{+}+2 \mathrm{e}^{-} \rightarrow \mathrm{H}_{2}
$$

Hydroxide ions that remain in the jet aperture may generate the observed positive nozzle current from ground to nozzle via the process:

$$
2 \mathrm{OH}^{-} \rightarrow \mathrm{H}_{2} \mathrm{O}+\mathrm{O}_{2}+2 \mathrm{e}^{-}
$$

However, we have no direct measurements to support this at the present time, and more complicated reactions may well be involved. It is observed that a single jet can be run for approximately 10 hours 
before there is a noticeable increase in diameter. The diameter increase may be due to electrochemical corrosion of the metal or it may be simple erosion.

Figure 4 shows mass spectrometer signals for an experiment using a 10 _m diameter jet with a constant flow rate of $0.5 \mathrm{~mL} / \mathrm{min}$. During the course of the experiment, the jet composition was varied from pure $\mathrm{H}_{2} \mathrm{O}$ to pure $\mathrm{D}_{2} \mathrm{O}$ and then back to $\mathrm{H}_{2} \mathrm{O}$. As charged ice builds up at the target electrode, the potential rises and drives increasing proton neutralization; consequently, the $\mathrm{H}_{2}{ }^{+}$signal increases. After 7 minutes, the composition of the jet was changed to pure $\mathrm{D}_{2} \mathrm{O}$; however, the response is delayed while the $\mathrm{D}_{2} \mathrm{O}$ travels to and fills the relatively large internal volume $(\sim 3 \mathrm{~mL})$ of the nozzle apparatus. The $\mathrm{H}_{2}^{+}$signal then decreases, while the $\mathrm{HD}^{+}$signal increases. As the mixing continues, the $\mathrm{HD}^{+}$signal reaches a maximum and then decreases while the $\mathrm{D}_{2}{ }^{+}$increases. After 31 minutes, the jet composition is returned to pure $\mathrm{H}_{2} \mathrm{O}$ and the process is shown to be entirely reversible.

The $\mathrm{H}_{2}{ }^{+}, \mathrm{D}_{2}{ }^{+}$, and $\mathrm{HD}^{+}$mass spectrometer signals are all characterized by intermittent spikes that confirm the production of molecular hydrogen at the jet target. The liquid nitrogen-cooled trap becomes coated with insulating layers of ice that separate what is essentially a charged icicle from the electrically grounded electrode. As the icicles collapse under gravity, or when the potential reaches a point where discharge through the ice becomes possible, a spike of hydrogen is observed. In other experiments, (not shown) hydrogen production closely followed the current measured at the target, including the spikes. Similarly, the same hydrogen production patterns were observed (without spikes) when the target electrode was warmed to avoid icicle buildup. These experiments rule out lightning bolt discharge in the charged ice as the only causative hydrogen production method.

Although the mass spectrometer uses a hot filament to generate electrons that are subsequently accelerated to $70 \mathrm{eV}$ and used to ionize sample molecules, it is not possible that the hydrogen signals observed in Figure 4 resulted either from cracking of water on this hot filament, or from fragmentation of water with $70 \mathrm{eV}$ electrons. Although, a certain percentage of the $\mathrm{H}_{2}$ background in the chamber originates from residual water that has been produced by these two mechanisms, they cannot account for the experimentally measured $\mathrm{H}_{2}$ and $\mathrm{D}_{2}$ signals. The $\mathrm{H}_{2} \mathrm{O}^{+}, \mathrm{HDO}^{+}$, and $\mathrm{D}_{2} \mathrm{O}^{+}$signals in Figure 4 show no observable change as the liquid composition varies from $\mathrm{H}_{2} \mathrm{O}$ to $\mathrm{D}_{2} \mathrm{O}$ and back. These flat signals imply that the marked changes found in the corresponding $\mathrm{H}_{2}^{+}, \mathrm{D}_{2}^{+}$, and $\mathrm{HD}^{+}$signals are not being produced at the hot filament or by fragmentation. There is roughly a three orders-of-magnitude difference between the electron ionization cross sections for production of $\mathrm{H}_{2} \mathrm{O}^{+}$and $\mathrm{H}_{2}^{+}$from $\mathrm{H}_{2} \mathrm{O}$ (similar for $\mathrm{D}_{2} \mathrm{O}$ ) ${ }^{23}$, so 
changes in the $\mathrm{H}_{2}{ }^{+}, \mathrm{D}_{2}{ }^{+}$, and $\mathrm{HD}^{+}$signals originating from fragmentation would unavoidably be associated with changes in the corresponding water signals. Similarly, isotopic changes in hydrogen that is formed at the hot filament would require corresponding isotopic changes in the water background. The flat $\mathrm{H}_{2} \mathrm{O}^{+}$, $\mathrm{D}_{2} \mathrm{O}^{+}$, and $\mathrm{HDO}^{+}$signals confirm that the condensable vapors from the jet chamber are effectively removed by the liquid nitrogen traps before entering the analysis chamber and, consequently, that the observed hydrogen signals originate from the gas produced in the jet chamber.

Figure 5 shows the $\mathrm{D}_{2} \mathrm{O}^{+}$and $\mathrm{D}_{2}^{+}$mass spectrometer signals as a function of time and at various jet velocities. After a build-up phase, at $\mathrm{t}=0$ the jet flow was reduced to $0.2 \mathrm{~mL} / \mathrm{min}$. Afterwards, the pure $\mathrm{D}_{2} \mathrm{O}$ jet was operated for 10 minutes at each of the flow rates indicated $(0.2,0.5,0.1,0.4,0.6,0.3 \mathrm{~mL} / \mathrm{min})$. Again, the $\mathrm{D}_{2}{ }^{+}$signal shows an irregular spiky pattern that indicates $\mathrm{D}_{2}$ formation as the charged icicles collapse and/or discharge to the grounded electrode surface. Despite the fact that unpredictable ice buildup and discharge give large spikes and depressions, there is an obvious step pattern in the $\mathrm{D}_{2}^{+}$signal as the flow rate is increased or decreased. Again, the $\mathrm{D}_{2} \mathrm{O}^{+}$signal remains constant throughout the experiment, indicating that the $\mathrm{D}_{2}$ is not formed in the analysis chamber.

The current at the nozzle is also plotted in Figure 5 (secondary axis). As expected, there is a clear relationship between the $\mathrm{D}_{2}^{+}$signal and the electrokinetic current. A plot of the average $\mathrm{D}_{2}^{+}$signal vs. average current for each flow rate gives a straight line, i.e. the hydrogen production exhibits the same (nearly quadratic) velocity dependence as the electrokinetic current.

While the present experiment was designed to measure hydrogen production from electrokinetic streaming currents, it also gives indirect insight into the nature of the unbiased Pt-water interface. In general, any contact between two materials with different chemical potentials (i.e. work functions) will induce charge transfer from one to the other until equilibrium is reached, and the chemical potentials become equal. Based on the sign of the measured streaming currents, as well as the observation that hydrogen gas is produced at the target, it is logical to conclude that hydroxide binds more favorably to the platinum surface, with partial charge transfer of electrons into the metal. While hydrated protons have recently been shown to preferentially adsorb to the water-air interface, ${ }^{24}$ hydroxide ions are known to preferentially adsorb to metal surfaces because of the very large induction interactions attending their large dielectric constants. ${ }^{12,13}$ Recent calculations indicate that hydroxide will contact adsorb to silver with charge transfer to the metal, while hydronium will adsorb in a solvated state. ${ }^{15,25}$ Hydrated protons in the diffuse layer would balance the charge from surface hydroxides; however, as the diffuse layer is sheared 
away from the surface, charge neutrality is lost and the corresponding potential increase drives the hydroxide ions to fully discharge into the metal. Consequently, electrons flow from the jet nozzle to ground and the streaming current is measured to be positive. As streaming currents at the jet target electrode and hydrogen production can measured irrespective of whether the nozzle is floating or grounded, it should be noted that hydroxide ions can back conduct through the liquid; however, unless necessary, this process is expected to be small due to the large resistance ( Tera_).

As hydrogen production was also observed from molybdenum aperatures, it is unlikely to be a result of any catalytic properties of the $\mathrm{Pt} / \mathrm{Ir}$ aperature. Gavis and Koszman indicate that there is little difference between electrokinetic charge generation rates between different metals. ${ }^{5}$

Considering the fact that most of the hydrogen produced at the grounded target electrode is pumped out of the chamber before detection, the efficiency of the energy conversion process could not be directly measured from the mass spectrometer signals. However, as hydrogen production is directly related to the measured current, the current can be used to obtain order of magnitude estimates for efficiency. For a 5 _m aperture flowing at $0.34 \mathrm{~mL} / \mathrm{min}$, the backing pressure is about $10 \mathrm{MPa}$ and the streaming current is about $180 \mathrm{nA}$. These numbers give a total mechanical power input of $0.057 \mathrm{~W}$ (flow rate pressure). Using the free energy of formation of liquid water and assuming that all the current generates hydrogen molecules, the chemical power for hydrogen production is about $2 \cdot 10^{-7} \mathrm{~W}$. Taking the ratio of chemical/mechanical power gives an efficiency of $\sim 4 \cdot 10^{-6}$.

The efficiency for this chemical process is thus quite low; however, there is also energy available in the associated electrical processes. The potential of electrically isolated nozzles (or targets) can be quite high, although the small currents (nA) make accurate potential measurements difficult to obtain. As a rough estimate for the potential, it was observed that isolated nozzles placed $\sim 0.5 \mathrm{~cm}$ from a grounded metal surface repeatedly arced to ground. Taking the breakdown voltage of air to be $3 \cdot 10^{6} \mathrm{~V} / \mathrm{m}$, the potential must be on the order of $\mathrm{kV}$. Using a moderate estimate of $1 \mathrm{kV}$ for the potential at the nozzle, the electrical power is found to be $\sim 2 \cdot 10^{-4} \mathrm{~W}$ with an efficiency of $\sim 3 \cdot 10^{-3}$.

It is possible that this electrical power can be directly utilized to produce hydrogen via electrolysis, this considerably improving the chemical efficiency. Furthermore, changes in the nozzle geometry to increase the surface area/volume ratio of the resulting liquid jet should improve the electrokinetic efficiency, as could the use of more exotic nozzle material. Optimization of the $\mathrm{pH}$ and ionic strength of the fluid, as well as temperature, flow values, and electrode bias will yield higher efficiency as well. Future experiments will 
focus on improving the chemical efficiency with different aperture materials and fluid compositions as well as by elucidating the mechanism by which anions are neutralized at the metal water-interface.

\section{Conclusion}

The electrokinetic streaming currents generated from water flowing through micron-sized Pt/Ir apertures can be modeled very well with Equation 11. As evident from the experimental data, as well as the fitting equation, the streaming current scales nearly quadratically with fluid velocity. The physical origin of the streaming current is the overlap of a hydrodynamic velocity gradient with the electrical double layer formed at the Pt-water interface. Although the present experiment did not give direct information about the Ptwater interface, the data indicate that the EDL is formed as hydroxide anions adsorb more favorably to the Pt surface than do hydronium cations, as supported by literature results. ${ }^{12,15}$ Pressure-driven flow creates a velocity profile that shears charge, the hydrated protons in this case, from the diffuse double layer and carries it out of the aperture. The pressure-induced separation of charge creates large electrical potentials that subsequently cause the ions to be neutralized at the grounded target electrode or in the nozzle. Neutralization (reduction) of the hydronium ions at the target electrode produces gaseous hydrogen molecules. It is suspected that oxygen is formed by oxidation in the jet aperture; however, we could not detect oxygen with the present experimental design. The hydrogen produced from electrokinetic streaming currents reversibly follows fluid composition as the jet is switched between $\mathrm{H}_{2} \mathrm{O}$ and $\mathrm{D}_{2} \mathrm{O}$. Hydrogen

production also follows jet velocity, with corresponding increases/decreases in hydrogen production as jet flow rate increases/decreases. As could be expected, there is a direct linear relationship between the streaming current and the amount of hydrogen produced. The present efficiency for hydrogen generation is ca. 10-6 but can be improved by several design considerations.

\section{Acknowledgements}

This research was funded by the Chemical Sciences, Geosciences and Biosciences Division, Office of Basic Energy Sciences, U.S. Department of Energy. We thank Kevin Wilson, Bruce Rude, and Jared Smith of Lawrence Berkeley National Lab, Chemical Sciences Division for their efforts in the initial phases 
of this study. We also thank Dorian Liepmann of the UCB Bioengineering department and Philip Marcus of the UCB Mechanical Engineering department for helpful conversations. 


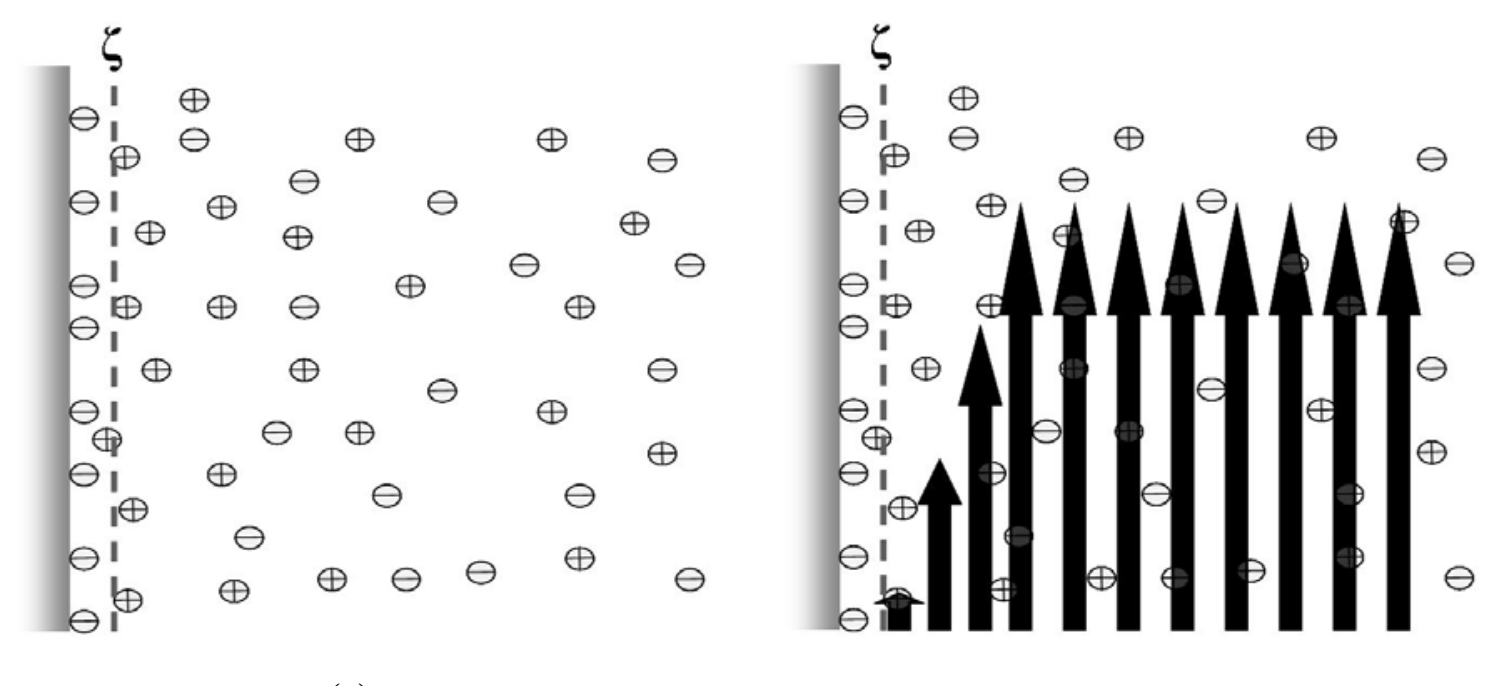

(a)

(b)

Figure 1: Metal-water interface. (a) Inhomogeneous charge distribution at a metal-water interface resulting from selective adsorption of hydroxide ions to the surface. (b) Overlap of charge distribution with the fluid velocity profile. The arrow height indicates positional liquid flow velocity in microjet nozzle. In both panels, the approximate position of the plane of shear is marked with the dashed line; the zeta potential (_) is the electrical potential at this plane with respect to the bulk liquid. Pressure-induced flow shears charge from the diffuse layer and leaves unbalanced negative charges at the metal-water interface, such that the emerging liquid jet is enriched in protons. 


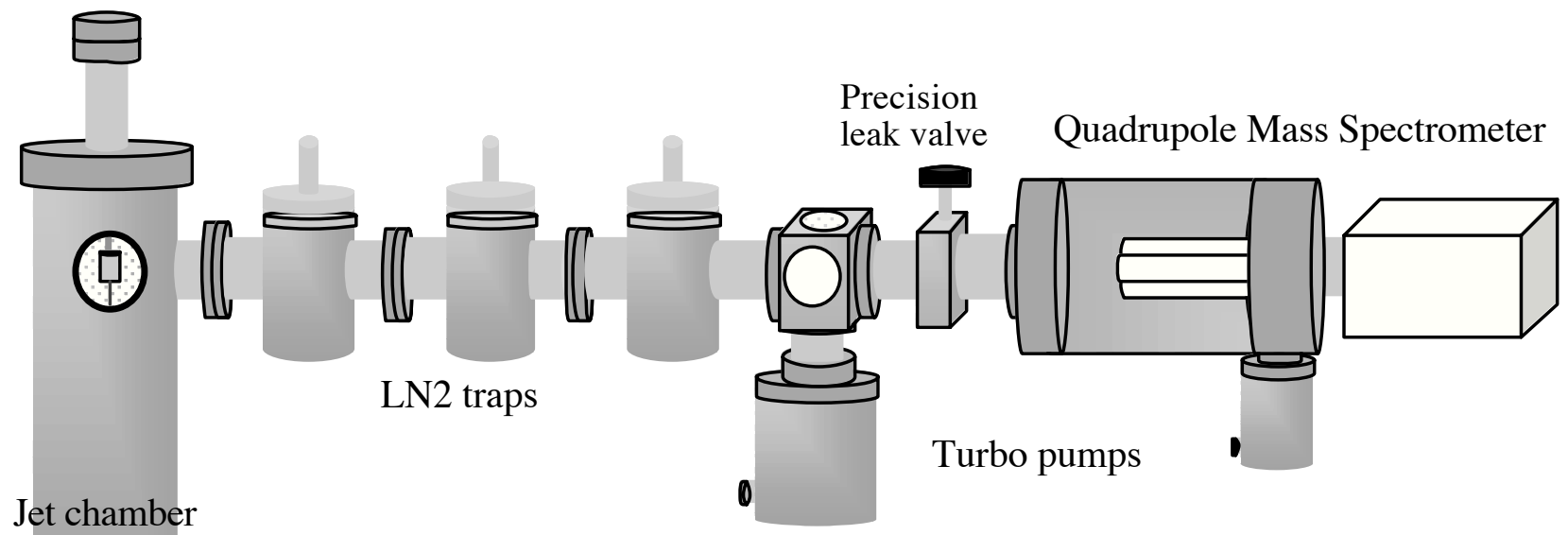

Figure 2: Diagram of experimental setup

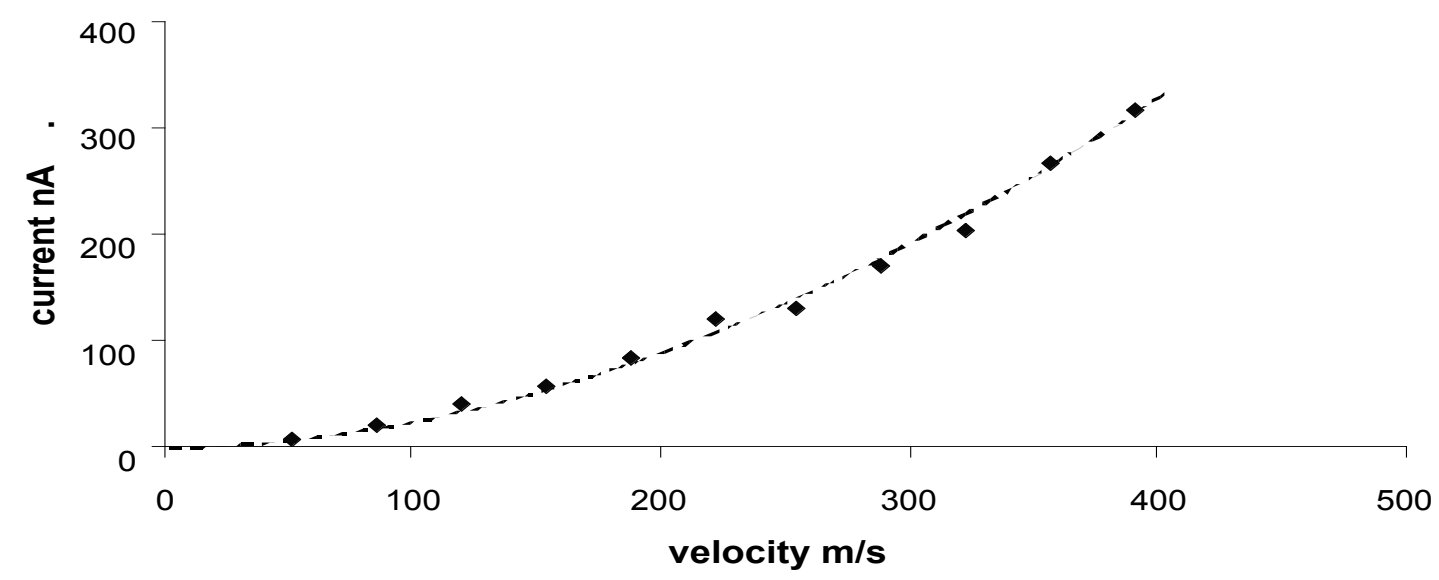

Figure 3: Streaming current as a function of jet velocity from a 5_m aperture. (_) experimental data; (--) streaming current model (Equation 11 in text) with $\_=-0.0275 \pm 0.0004 \mathrm{~V}\left(\mathrm{R}^{2}=0.9933\right)$ The data confirm a nearly quadratic increase in current with fluid velocity, as predicted from theory. 


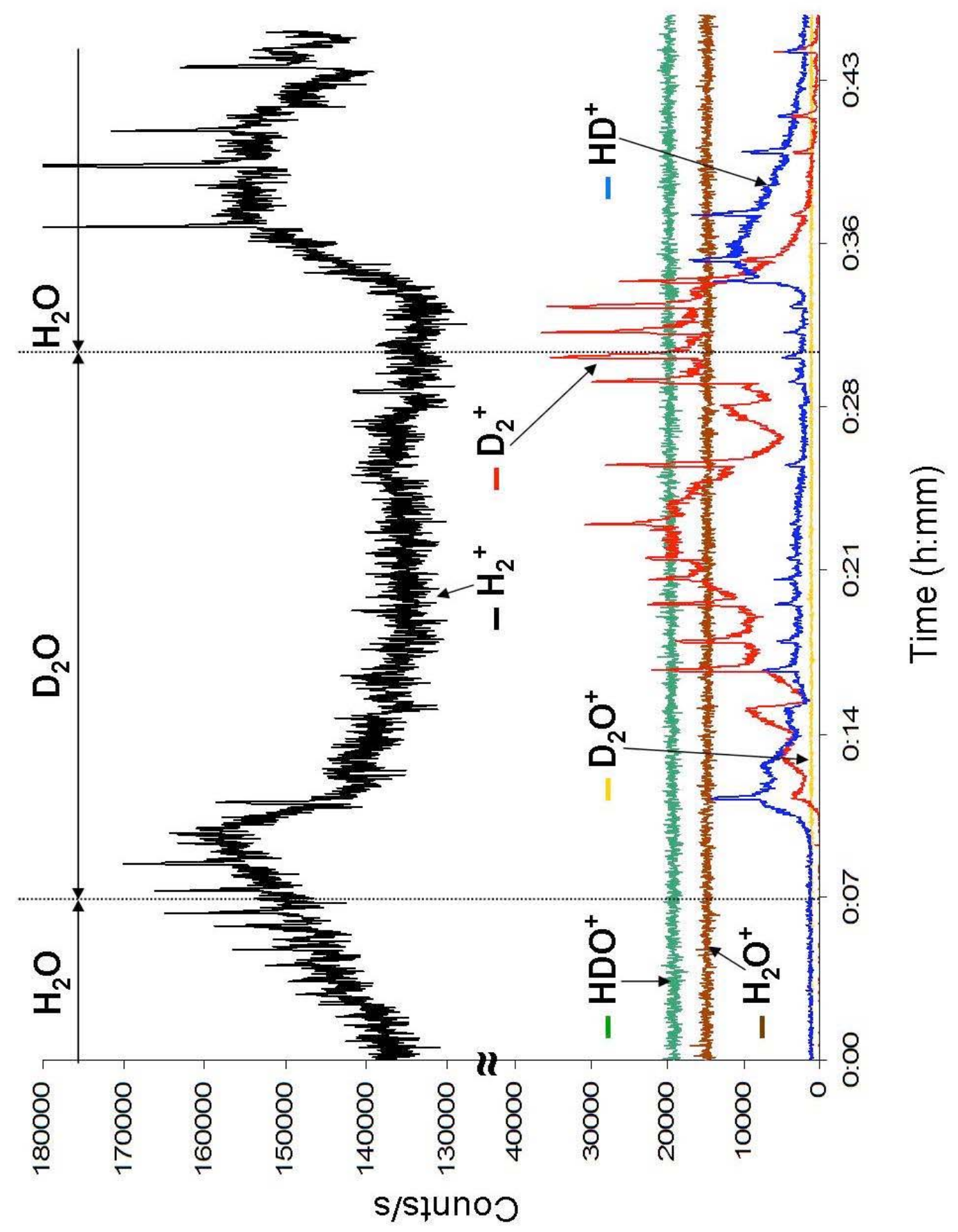

Figure 4: Mass spectrometer signals for hydrogen and water isotopes as liquid jet composition is switched from $\mathrm{H}_{2} \mathrm{O}(0-7 \mathrm{~min})$ to $\mathrm{D}_{2} \mathrm{O}$ (7-31 min) and back to $\mathrm{H}_{2} \mathrm{O}$ (31-47 min). Black $-\mathrm{H}_{2}{ }^{+}$, Red $-\mathrm{D}_{2}{ }^{+}$, Blue $-\mathrm{HD}^{+}$, Brown $-\mathrm{H}_{2} \mathrm{O}^{+}$, Yellow $-\mathrm{D}_{2} \mathrm{O}^{+}$, Green $-\mathrm{HDO}^{+}$Data were collected with a $10 \_\mathrm{m}$ diameter jet flowing at $0.5 \mathrm{ml} / \mathrm{min}$. Note that isotopic hydrogen signals follow jet composition while the oxygencontaining water signals are invariant. 


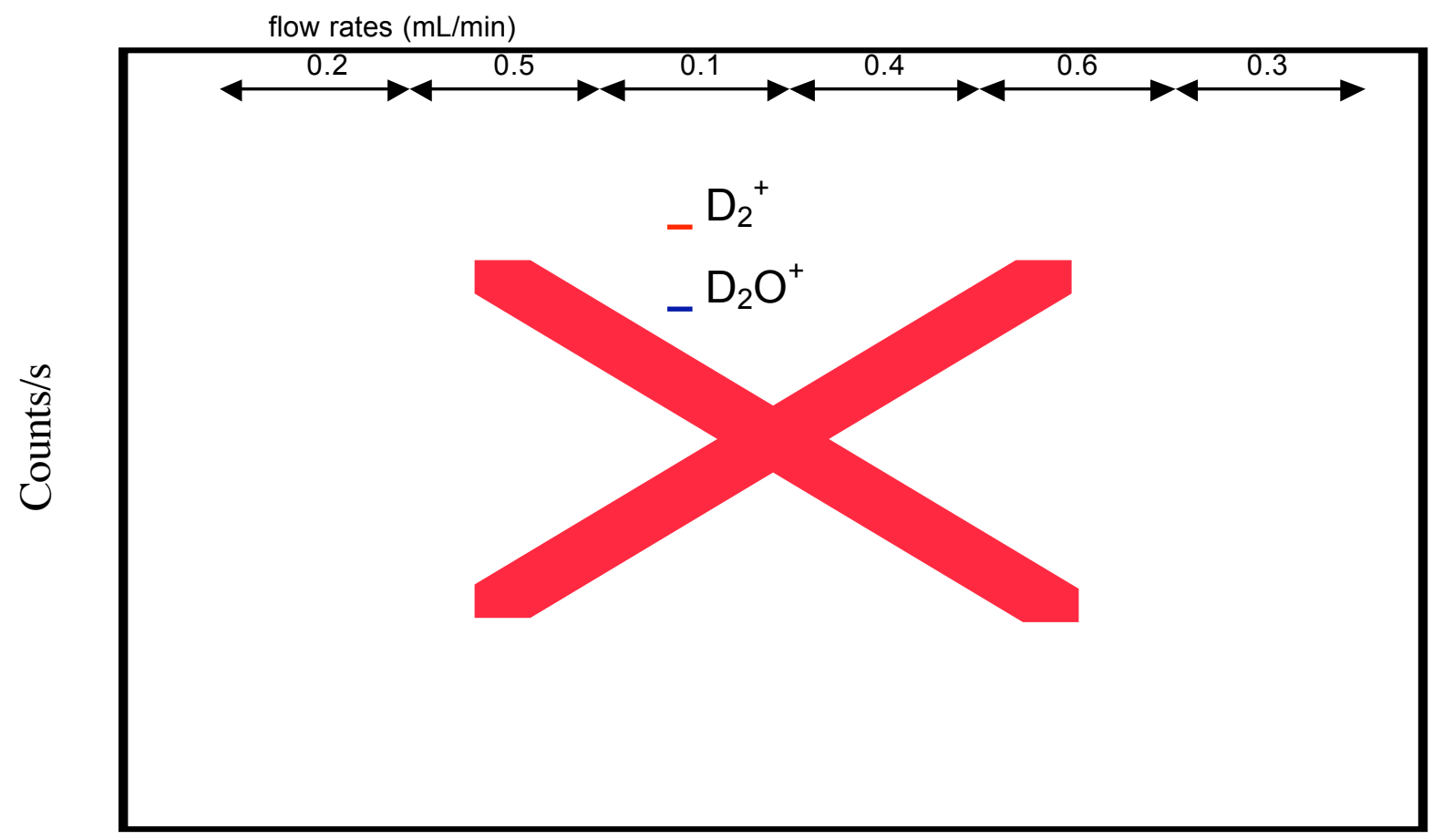

Time (h:mm)

Figure 5: $\mathrm{D}_{2}{ }^{+}$and current measurements from a $10 \_\mathrm{m}$ jet at varying jet velocities (flow rates indicated at the top). Primary axis: $\mathrm{D}_{2}^{+}$(red) and $\mathrm{D}_{2} \mathrm{O}^{+}$(blue) mass spectrometer signals. Note the $\mathrm{D}_{2}^{+}$signal changes with jet velocity while the $\mathrm{D}_{2} \mathrm{O}^{+}$is invariant. Secondary axis: electrokinetic current $\left(\_\right)$measured at nozzle. The $\mathrm{D}_{2}^{+}$signal is found to be proportional to the current, i.e. both scale nearly quadratically on flow velocity. 
References

(1) Service, R. F. Science 2004, 305, 958.

(2) Stojic, D. L.; Marceta, M. P.; Sovilj, S. P.; Miljanic, S. S. Journal of Power Sources 2003, 118,315 .

(3) Davies, J. T.; Rideal, E. K. Interfacial Phenomena; Academic Press: New York, 1961.

(4) Lyklema Fundamentals of Interface and Colloid Science Vol. 2, 1995; Vol. 2.

(5) Gavis, J.; Koszman, I. Journal of Colloid Science 1961, 16, 375.

(6) Rice, C. L.; Whitehead, R. Journal of Physical Chemistry 1965, 69, 4017.

(7) Dolezalek, F. Chemistry and Industry 1913, 36, 33.

(8) Klinkenberg, A.; Van Der Minne, J. L. Electrostatics in the Petroleum Industry-The Prevention of Explosion Hazards; Elsevier: New York, 1958.

(9) Yang, J.; Lu, F. Z.; Kostiuk, L. W.; Kwok, D. Y. Journal of Micromechanics and Microengineering 2003, 13, 963.

(10) Olthuis, W.; Schippers, B.; Eijkel, J.; van den Berg, A. Sensors and Actuators B-Chemical 2005, 111,385 .

(11) vanderHeyden, F. H. J.; Bonthuis, D. J.; Stein, D.; Meyer, C.; Dekker, C. Nano Lett. 2007, 7,1022

(12) Bruesch, P.; Christen, T. Journal of Applied Physics 2004, 95, 2846.

(13) Magnussen, O. M. Chemical Reviews 2002, 102, 679.

(14) Service, R. F. Science 2007, 315, 172.

(15) Patrito, E. M.; Paredes-Olivera, P. Surface Science 2003, 527, 149.

(16) Holstein, W. L.; Hayes, L. J.; Robinson, E. M. C.; Laurence, G. S.; Buntine, M. A. Journal of Physical Chemistry B 1999, 103, 3035. 
(17) Delgado, A. V.; Gonzalez-Caballero, F.; Hunter, R. J.; Koopal, L. K.; Lyklema, J. Journal of Colloid and Interface Science

Elkin 06, International Electrokinetics Conference, June 25-29, Nancy, France 2007, 309, 194.

(18) White, F. M. Fluid Mechanics, 4 ed.; WCB Mcgraw-Hill, 1999.

(19) Hignett, E. T.; Gibbings, J. C. Journal of Electroanalytical Chemistry 1968, 16, 239.

(20) Rouse, H.; Howe, J. W. Basic Mechanics of Fluids; John Wiley \& Sons, Inc.: Londeon, 1953.

(21) Faubel, M.; Steiner, B. Berichte Der Bunsen-Gesellschaft-Physical Chemistry Chemical Physics 1992, 96, 1167.

(22) Geissler, P. L.; Dellago, C.; Chandler, D.; Hutter, J.; Parrinello, M. Science 2001, 291, 2121.

(23) Straub, H. C.; Lindsay, B. G.; Smith, K. A.; Stebbings, R. F. Journal of Chemical Physics 1998, 108, 109.

(24) Petersen, P. B.; Saykally, R. J. Journal of Physical Chemistry B 2005, 109, 7976.

(25) Olivera, P. P.; Ferral, A.; Patrito, E. M. Journal of Physical Chemistry B 2001, 105, 7227. 